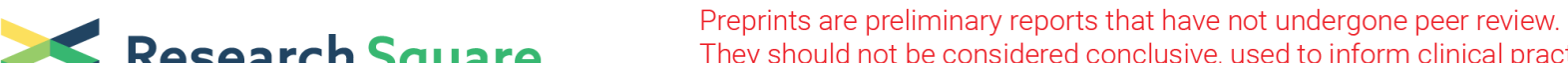 Research Square They should not be considered conclusive, used to inform clinical practice, or referenced by the media as validated information. \\ Relationship between the Timing of Pregnancy \\ Termination and Pregnancy Outcome and the Short- term Outcome of Infants with a Decreased Cerebroplacental Ratio: A Retrospective Cohort \\ Study
}

\section{Bimei Hu}

Lianyungang Branch of Traditional Chinese Medicine, Jiangsu Union Technical Institute

\section{Zhaoer Yu}

Department of obstetrics $₫$ Women's Hospital of Nanjing Medical University $\mathbb{N}$ Nanjing Maternity and Child Health Care Hospital

\section{Mingming Gao}

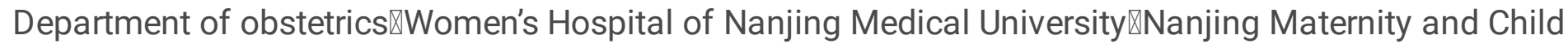
Health Care Hospital

\section{Dan Yao}

Department of obstetrics $₫$ Women’s Hospital of Nanjing Medical University $\mathbb{N}$ Nanjing Maternity and Child Health Care Hospital

\section{Yating Qian}

Department of obstetrics $₫$ Women’s Hospital of Nanjing Medical University $₫$ Nanjing Maternity and Child Health Care Hospital

\section{Hongjuan Ding}

Department of obstetrics $₫$ Women’s Hospital of Nanjing Medical University $\mathbb{N}$ Nanjing Maternity and Child Health Care Hospital

\section{Ruizhe Jia ( $\square$ jiaruizhe2016@163.com )}

Department of obstetrics $₫$ Women’s Hospital of Nanjing Medical University $\mathbb{N}$ Nanjing Maternity and Child Health Care Hospital

\section{Research Article}

Keywords: fetal umbilical artery resistance, fetal hypoxia, redistribute fetal blood flow, middle cerebral artery, cerebroplacental rate (CPR)

Posted Date: January 27th, 2021

DOI: https://doi.org/10.21203/rs.3.rs-148209/v1 
License: (c) (i) This work is licensed under a Creative Commons Attribution 4.0 International License. Read Full License 


\section{Abstract}

Background: Increased fetal umbilical artery resistance can cause fetal hypoxia, redistribute fetal blood flow, dilate the middle cerebral artery, and reduce the cerebroplacental rate (CPR). CPR $<1$ is a danger sign of fetal hypoxia. Choosing the appropriate time for terminating a pregnancy with the most favorable pregnancy outcome and the short-term outcome of infants, based on the abnormal performance of the cord blood flow, is crucial.

Methods: A total of 114 pregnant women with CPR $<1$ who gave birth at Obstetrics and Gynecology Hospital Affiliated to Nanjing Medical University from January 2015 to December 2019 were analyzed retrospectively. The age, height, and weight of the aforementioned pregnant women, as well as the gestational age when CPR $<1$ occurs for the first time, were recorded. The women were divided into two groups, based on pregnancy termination: the immediately terminated pregnancy group and the continued pregnancy group. The pregnancy outcomes, neonatal outcomes, and short-term outcome of the surviving infants in both groups were recorded and compared.

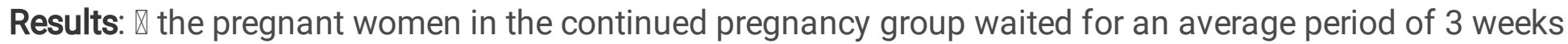
prior to the termination of pregnancy beyond 32 weeks. The average weight of the newborn was $1429 \mathrm{~g}$, which was significantly lower than that of the average newborn at the corresponding gestational age. In the continued pregnancy group, intrauterine death was reported in 18 cases (40.9\%) and intrauterine death of one fetus in a twin pregnancy was reported in 2 cases. Intrauterine death occurred 2-33 day after $\mathrm{CPR}<1$ ( $12.8 \mathrm{~d}$ on average) as determined. $\otimes$ the incidence rates of neonatal asphyxia, neonatal pneumonia, neonatal sepsis, and neonatal anemia in the continued pregnancy group were significantly

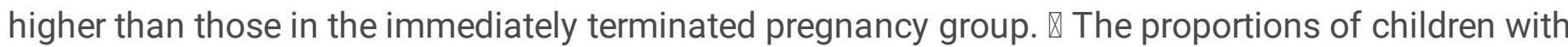
autism spectrum disorder, motor development retardation, and growth retardation were slightly higher in the continued pregnancy group than in the immediately terminated pregnancy group; however, the difference was not statistically significant.

Conclusion: Intrauterine hypoxia is considered severe when CPR $<1$. In this case, a cesarean section should be conducted immediately, and waiting may cause an increased risk of neurodevelopment disorder in children.

\section{Background}

The fetal umbilical artery is the channel connecting the fetus to the mother. Any factor that affects blood flow in the umbilical artery and increases its circulatory resistance may cause fetal hypoxia. At the same time, as an adaptation mechanism to hypoxia, the blood flow of the fetus will be redistributed, which is beneficial to vital organs, such as the heart and the brain(1). Cerebral vasodilatation is considered an early protective self-regulatory mechanism, referred to as the "brain-sparing effect"(2). The pulsatility index $(\mathrm{PI})$ of the middle cerebral artery (MCA)/uterine artery (UA) ratio-the cerebroplacental ratio $(\mathrm{CPR})<$ $1-$ can be used as a predictive indicator of the brain-sparing effect of the fetus(3). However, recent 
research results indicate that CPR, which is often used clinically to evaluate cord blood flow redistribution, may not completely achieve the brain-sparing effect(4). Poor neonatal outcomes, decreased neonatal brain volume, and increased risk of adverse neurocognitive outcomes in early childhood are related to fetal cerebral vasodilation(5).

The ratio of umbilical blood flow is an important indicator of fetal intrauterine safety. Absent enddiastolic flow (AEDF) and reversed end-diastolic flow (REDF) are currently regarded as danger signs of intrauterine fetal death $(6,7) ; \mathrm{CPR}<1$ is also a danger sign of fetal hypoxia(3). Both aforementioned conditions are prone to high perinatal mortality. Cesarean section is recommended as soon as possible. However, if the gestational age is inadequate at this time, then the premature babies also have risk factors, such as various complications, low survival rate, and unknowable long-term outcomes, which can result in an increased burden to the family and society. The method of selecting the time for the most favorable pregnancy outcome and the short-term outcome of infants to terminate the pregnancy, based on abnormal cord blood flow, is particularly important.

In this study, color Doppler ultrasound was used to examine the changes in blood flow in the fetal umbilical artery and MCA. Pregnant women with $\mathrm{CPR}<1$ were selected. Their pregnancy outcome, neonatal outcome, and short-term outcome of infants was followed up. The correlation was determined between different time frames of termination of pregnancy and pregnancy outcome, neonatal outcome, and short-term outcome of infants. A theoretical basis was provided for the clinical selection of the appropriate timing of pregnancy termination.

\section{Materials And Methods}

\section{Patient, study design, and clinical data}

A retrospective analysis was conducted on 114 pregnant women with CPR $<1$ who gave birth at the Obstetrics and Gynecology Hospital Affiliated to Nanjing Medical University from January 2015 to December 2019. Fetal chromosomal abnormalities were excluded. The study was approved by the ethics committee of the hospital with the informed consent of the selected patients and their families.

The ages, height, weight and the gestational age when CPR $<1$ occurred for the first time of the aforementioned pregnant women were recorded. The patients were divided into two groups, based on whether the pregnancy was terminated immediately: immediately terminated pregnancy group and continued pregnancy group. The pregnancy outcome, neonatal outcome, and short-term outcome of infants in the two groups were recorded and compared. The Medical Ethics Committee of Nanjing Maternity and Child Health Care Hospital has approved this study, and the registry number is $2019 \mathrm{KY}$ 046. All the experiment protocol for involving humans was Performed in accordance to guidelines of national

\section{Instruments and examination methods}


Philips iU22, Siemens S2000, GE E8, Alokas SSD-5500, and other color Doppler ultrasonic imagers were used. The probe frequency was set to 3.5-5 MHz. The pregnant woman was placed in a supine position and abdominal radiography was performed. Fetal growth indexes, such as the double apex diameter, head circumference, abdominal circumference, and femur length, were determined by two-dimensional ultrasound. The placenta and amniotic fluid were also observed.

Color Doppler ultrasound was conducted to detect blood flow. All hemodynamic tests were performed while the fetus was in the resting state, without breathing or movement, and the instrument settings used to check the fetus were kept constant. The ultrasound beam was set parallel as possible to the direction of blood flow. In the measurement of the umbilical artery (UA), a free segment of the umbilical cord in the amniotic fluid was selected, and a cross-section of MCA was taken from the biparietal plane to the level of the sphenoid wing. Examinations were conducted when the circle of Willis was fully displayed. The sampling volume was placed in the near-field MCA immediately adjacent to the origin of the internal carotid artery and was not mixed with the internal carotid artery blood flow. When the blood flow in UA and MCA had 5 consecutive spectra with similar shapes and regular rhythms, the hemodynamic parameters to be recorded were as follows: the peak systolic velocity, end-diastolic blood flow velocity, average velocity of blood flow, pulsation index, and resistance index. Loss or a reversal of the enddiastolic blood flow was recorded.

Methods of assessment of short-term outcomes of infants: General movements (GMs) was used regularly to assess whether a motor developmental delay was observed in children. The M-CHAT autism spectrum disorder scale was used to detect the presence of the disorder. Child development was assessed using the Gesell scale. The height and weight of children were measured regularly to assess for malnutrition (Malnutrition was defined as a child's age-specific weight/height below the median minus 2 standard deviations from the reference population standard for the same age and sex). Regular screening was conducted to assess for language barrier, visual impairment, and hearing impairment.

\section{Statistical analysis}

Data were statistically analyzed using SPSS 26.0. The data measured were expressed as $x \Downarrow \pm \mathbf{S}$, and Student's t-test was used. The data enumerated were expressed as $\mathrm{n}(\%)$, and intergroup comparison was performed using the Chi-square test.

\section{Results}

A total of 114 pregnant women with $\mathrm{CPR}<1$ were included in this study. Umbilical artery resistance increased for the first time in 20-401/7 weeks (average $31.50 \pm 4.24$ weeks) gestation, and CPR $<1$ first occurred in 21-401/7 weeks (average 32.52 \pm 4.03 weeks) of gestation, with an interval time of 0-50 d (average $6.65 \pm 11.38$ days). A total of 13 patients had CPR $<1$ and AEDF. 
Seven cases were lost to follow-up, whereas 107 cases remained. The 107 pregnant women were divided into two groups, based on whether the pregnancy was terminated immediately: the terminated pregnancy group (63 cases) and the continued pregnancy group (44 cases) (Fig. 1). The gestational age when CPR $<1$ and the weight of the newborn (if twins, the recorded newborn weight was the weight of the fetus with $\mathrm{CPR}<1$ after delivery) in the terminated pregnancy group were significantly greater than those in the continued pregnancy group $(P<0.05)$; no statistically significant differences in the age, height, and weight of women were found between the two groups $(P>0.05)$ (Table 1$)$. The analysis of pregnancy outcomes in the two groups showed intrauterine fetal death in $18(40.9 \%)$ of the 44 patients who opted to continue their pregnancies, and 2 cases were twin pregnancies in which one fetus died in utero, the time leading to the occurrence of fetal death in utero was $21-33 d$ (an average of 12.8 days) after CPR $<1$ was detected. In addition, no significant difference in pregnancy complications, including preeclampsia, fetal growth restriction, intrahepatic cholestasis of pregnancy, oligohydramnios, and twin pregnancy was found $(p>0.05)$ (Table 2).

Table 1

Basic characteristics of patients by group

\begin{tabular}{|llll|}
\hline Patient characteristics & $\begin{array}{l}\text { Immediately terminated } \\
\text { pregnancy group(n= 63) }\end{array}$ & $\begin{array}{l}\text { Continued pregnancy } \\
\text { group }(\mathbf{n}=\mathbf{4 4})\end{array}$ & $\boldsymbol{P}$ \\
\hline Age $(\mathrm{y})$ & $31.27 \pm 4.80$ & $29.73 \pm 4.49$ & 0.096 \\
\hline Height $(\mathrm{cm})$ & $160.86 \pm 4.40$ & $161.93 \pm 5.26$ & 0.254 \\
\hline Weight $(\mathrm{kg})$ & $71.92 \pm 10.38$ & $69.84 \pm 9.66$ & 0.296 \\
\hline $\begin{array}{l}\text { Gestational week when CPR } \\
<1 \text { occurs (w) }\end{array}$ & $34.66 \pm 3.04$ & $29.85 \pm 3.48^{\star}$ & 0.000 \\
\hline Pregnancy week (w) & $34.66 \pm 3.04$ & $32.32 \pm 3.83^{\star}$ & 0.001 \\
\hline Newborn weight (g) & $1927.21 \pm 716.67$ & $1429.12 \pm 723.40^{\star}$ & 0.002 \\
\hline$* P<0.05$ & & & \\
\hline
\end{tabular}


Table 2

Pregnancy outcome by group

\begin{tabular}{|llll|}
\hline & $\begin{array}{l}\text { Immediately terminated } \\
\text { pregnancy group (\%) } \\
(\mathbf{n = 6 3 )}\end{array}$ & $\begin{array}{l}\text { Continued pregnancy } \\
\text { group (\%) } \\
(\mathbf{n}=\mathbf{4 4})\end{array}$ & $\boldsymbol{P}$ \\
\hline Fetal death in the uterus & 0 & $18(40.9)^{\star}$ & 0.000 \\
\hline Preeclampsia & $18(28.6)$ & $17(38.6)$ & 0.275 \\
\hline Fetal growth restriction & $37(58.7)$ & $27(61.4)$ & 0.785 \\
\hline $\begin{array}{l}\text { Intrahepatic cholestasis of } \\
\text { pregnancy }\end{array}$ & $1(1.6)$ & 0 & 0.351 \\
\hline Oligohydramnios & $13(20.6)$ & $6(13.6)$ & 0.982 \\
\hline Twin pregnancy & $13(20.6)$ & $9(20.5)$ & \\
\hline$* P<0.05$ & & & \\
\hline
\end{tabular}

Patients who choose to continue pregnancy were intramuscularly injected with dexamethasone (6 mg q12h, 4 times) to promote fetal lung maturity treatment (8), strengthen monitoring during treatment, and evaluate the color Doppler ultrasound results regularly.

During the waiting period, 14 patients with CPR $<1$ continued to develop AEDF, with an interval of 1-24 $d$ (average $8.8 \mathrm{~d}$ ); only 3 patients with AEDF changed to CPR $<1$; no REDF was detected in the patients. Only 2 patients had abnormal a-wave venous catheters, and intrauterine death occurred within a short period during follow-up.

Intrauterine death occurred in $18(40.9 \%)$ of the 44 cases in the continued pregnancy group. Among them, 17 cases retained CRP $<1$ or further developed into AEDF; only 1 returned to normal CRP, and the fetus died after reexamination with $\mathrm{CRP}<1$. The 26 patients who gave birth to live fetuses were divided into the following four groups, based on changes in condition: Group A, 4 patients $(9.1 \%)$ restored normal CRP and normal cord blood flow continued to be monitored until full-term delivery; Group B, 11 patients $(25.0 \%)$ waited for a short period ( $2-5 \mathrm{~d}$ ) and retained CRP $<1$ for cesarean section; Group C, 5 patients $(11.4 \%)$ had persistent CRP $<1$ and underwent cesarean section due to abnormal fetal heart rate (the pregnancy time was expected to exceed 3 weeks); and Group D, 6 patients $(13.6 \%)$ recovered normal CRP within a short period, but the blood flow in the umbilical artery remained abnormal. Subsequently, owing to abnormal fetal heart rate, $\mathrm{CRP}<1$, or other obstetric factors, cesarean section was conducted on the patients.

\section{Comparison of neonatal outcomes}


Among the 107 cases that could be followed up, 89 cases remained, including 20 cases of twins, and 18 cases of fetal death in the uterus were excluded. This study only analyzed the neonatal outcomes of fetuses with abnormal blood flow in the artery after delivery. Therefore, 63 cases with 13 cases of twins remained in the immediately terminated pregnancy group 0 and 26 cases with 7 cases of twins were found in the continued pregnancy group. The outcome analysis of the two groups of newborns is listed in Table 3. The incidence rates of neonatal asphyxia, neonatal pneumonia, neonatal sepsis, and neonatal anemia in the continued pregnancy group were significantly higher than those in the immediately terminated pregnancy group $(P<0.05)$. The incidence rates of neonatal respiratory distress syndrome and bronchopulmonary dysplasia were higher in the continued pregnancy group than in the immediately terminated pregnancy group; however, the difference was not statistically significant $(P>0.05)$. Intensive care, neonatal death, intracranial hemorrhage, necrotizing enterocolitis, gastrointestinal hemorrhage, hyperbilirubinemia, hypoglycemia, small for gestational age, and feeding intolerance had similar incidences in both neonate groups $(P>0.05)$. 
Table 3

Neonatal outcome by study group

\begin{tabular}{|llll|}
\hline & $\begin{array}{l}\text { Immediately terminated } \\
\text { pregnancy group (\%) } \\
(\mathbf{n = 6 3 )}\end{array}$ & $\begin{array}{l}\text { Continued } \\
\text { pregnancy group (\%) } \\
(\mathbf{n}=44)\end{array}$ & $P$ \\
\hline Newborn in ICU & $49(77.8)$ & $22(84.6)$ & 0.465 \\
\hline Neonatal death & $6(9.5)$ & $5(19.2)$ & 0.362 \\
\hline Fatal dysplasia & 1 & 0 & \\
\hline $\begin{array}{l}\text { Intrauterine death of one fetus in } \\
\text { a twin pregnancy }\end{array}$ & 2 & 4 & 0.034 \\
\hline Neonatal asphyxia & $2(3.2)$ & $5(19.2)^{\star}$ & 0.992 \\
\hline Neonatal intracranial hemorrhage & $29(46.0)$ & $12(46.2)$ & 0.115 \\
\hline $\begin{array}{l}\text { Neonatal respiratory distress } \\
\text { syndrome }\end{array}$ & $16(25.4)$ & $11(42.3)$ & 0.292 \\
\hline Neonatal necrotizing enterocolitis & 0 & $1(3.8)$ & 0.968 \\
\hline Neonatal gastrointestinal bleeding & $3(4.8)$ & $2(7.7)$ & 0.011 \\
\hline Neonatal pneumonia & $1(1.6)$ & $5(19.2)^{\star}$ & 0.041 \\
\hline Neonatal sepsis & $5(7.9)$ & $7(26.9)^{*}$ & 0.962 \\
\hline Neonatal hyperbilirubinemia & $27(42.9)$ & $11(42.3)$ & 1 \\
\hline Neonatal hypoglycemia & $5(7.9)$ & $2(7.7)$ & 0.003 \\
\hline Neonatal anemia & $6(9.5)$ & $10(38.5)^{*}$ & 0.150 \\
\hline Bronchopulmonary dysplasia & 0 & $2(7.7)$ & 0.802 \\
\hline Small for gestational age & $19(30.1)$ & $9(34.6)$ & 1 \\
\hline Feeding intolerance & $12(19.0)$ & & \\
\hline * P<0.05 & & & \\
\hline
\end{tabular}

\section{Analysis of short-term outcome of infants}

An early prognosis analysis of the remaining 73 children, excluding 11 cases of neonatal deaths and 5 children lost to follow-up, is listed in Table 3. The proportion of children with autism spectrum disorder in the continued pregnancy group was higher than that in the immediate termination group; however, the difference was not statistically significant $(P>0.05)$. There was no significant difference in the rate of delayed motor development and delayed motor development between the group of continuing pregnancy 
and the group of immediate termination $(P>0.05)$. The rates of malnutrition, language impairment, visual impairment, and hearing impairment in both groups were similar $(P=1)$ (Table 4).

We further analyzed the long-term prognosis of the children in each subgroup of the continuing pregnancy group; the children in Groups $B$ and $C$ had higher rates of poor long-term prognosis than those in Groups A and D (Table 5).

Table 4

Long-term prognosis of children by study group

\begin{tabular}{|llll|}
\hline & $\begin{array}{l}\text { Immediately terminated pregnancy } \\
\text { group (\%) } \\
(\mathbf{n = 5 5 )}\end{array}$ & $\begin{array}{l}\text { Continued pregnancy } \\
\text { group (\%) } \\
(\mathbf{n = 1 8 )}\end{array}$ & $\boldsymbol{P}$ \\
\hline $\begin{array}{l}\text { Autism Spectrum } \\
\text { Disorder }\end{array}$ & 0 & $2(11.1)$ & 0.094 \\
\hline Motor retardation & $19(34.5)$ & $8(44.4)$ & 0.575 \\
\hline Growth retardation & $13(23.6)$ & $6(33.3)$ & 0.537 \\
\hline $\begin{array}{l}\text { Language } \\
\text { impairment }\end{array}$ & $9(16.4)$ & $3(16.7)$ & 1 \\
\hline Visual impairment & $2(3.6)$ & $1(5.6)$ & 1 \\
\hline Hearing impaired & $1(1.9)$ & 0 & 1 \\
\hline Malnutrition & $20(36.4)$ & $7(38.9)$ & 1 \\
\hline
\end{tabular}

Table 5

Long-term prognosis of children in each subgroup of the continued pregnancy group

\begin{tabular}{|lllll|}
\hline & $\begin{array}{l}\text { Group A (\%) } \\
(\mathbf{n = 4 )}\end{array}$ & $\begin{array}{l}\text { Group B (\%) } \\
(\mathbf{n = 9})\end{array}$ & $\begin{array}{l}\text { Group C (\%) } \\
(\mathbf{n = 2})\end{array}$ & $\begin{array}{l}\text { Group D (\%) } \\
(\mathbf{n}=\mathbf{3})\end{array}$ \\
\hline Autism Spectrum Disorder & 0 & $2(22.22)$ & 0 & 0 \\
\hline Motor retardation & $1(25.00)$ & $6(66.67)$ & $1(50.00)$ & 0 \\
\hline Growth retardation & 0 & $5(55.56)$ & $1(50.00)$ & 0 \\
\hline Language impairment & 0 & $2(22.22)$ & $1(50.00)$ & 0 \\
\hline Visual impairment & 0 & $1(11.11)$ & 0 & 0 \\
\hline Malnutrition & 0 & $5(55.56)$ & $2(100.00)$ & 0 \\
\hline
\end{tabular}

\section{Discussion}


The UA is the channel for nutrient exchange between the fetus and the mother. Color Doppler ultrasound is typically used to check the blood flow of the fetal umbilical artery. The PI of the MCA is normally higher than that of the umbilical artery; however, when the umbilical blood flow resistance progressively increases, fetal hypoxia gradually increases, and a brain-sparing effect occurs, effectively redistributing oxygen and nutrients to vital organs (the brain, heart, and adrenal glands), manifested by increased blood flow in the MCA during systole, decreased PI and S/D ratios, even lower than the PI of cord blood flow, the $\mathrm{PI}$ of the MCA/UA-CPR < 1(3). When an abnormal increase in umbilical blood flow resistance is observed, the blood flow signal of the umbilical artery at the end of diastole decreases and even disappears (AEDF), leading to reflux (REDF). These occurrences and CPR $<1$ are danger signs of intrauterine fetal death(9).

In clinical practice, $\mathrm{CPR}<1$ is a widely recognized danger sign of fetal hypoxia and intrauterine fetal death. If the aforementioned abnormalities are observed during umbilical blood flow monitoring and the fetus can survive in vitro, except for fetal malformations and chromosomal abnormalities, pregnancy is mostly actively terminated by cesarean section to free the fetus of poor intrauterine environment as soon as possible for in vitro treatment. In this study, patients in the immediately terminated pregnancy group had a longer gestational week when CPR $<1$, with 34 weeks being the average. Considering the probability of fetal survival after birth and improved long-term prognosis, the patients opted for immediate termination of pregnancy. In the continuing pregnancy group, the gestational age when CPR < 1 occurred was relatively short, averaging 29 weeks only. Concerned about poor survival ability after birth, the patient chose to continue the pregnancy, waiting for an average of 3 weeks; beyond 33 weeks, pregnancy was terminated, although the newborn weight was still significantly lower than the average weight for the corresponding gestational age. After the occurrence of $\mathrm{CPR}<1$, the intrauterine environment of the fetus remained significantly poor, and the intrauterine growth rate was slow.

In the current study, the average gestational age when the umbilical blood flow resistance increased for the first time was 31.50 weeks; the average gestational age for the first occurrence of CPR $<1$ was 32.52 weeks; and the average interval was $6.65 \mathrm{~d}$. Therefore, after the umbilical blood flow resistance increased, color Doppler ultrasound was reviewed at least weekly to eventually determine the change in the umbilical blood flow. Intrauterine death was reported in 18 (40.9\%) cases of continued pregnancy. The time of occurrence of fetal death in the uterus was $2-33 \mathrm{~d}$ after CPR $<1$ was determined (average $12.8 \mathrm{~d}$ ). Studies have shown that the time interval from inspection to AEDF to fetal death is $1-14 \mathrm{~d}$, with an average of $6.3 \mathrm{~d}(10)$. Therefore, after determining CPR $<1$ or AEDF in the umbilical blood flow and MCA blood flow is determined, attention should be directed toward strengthening monitoring to promptly detect abnormal fetal heart rates.

Studies have shown that CPR reduction is manifested before serious UA abnormalities occur(11). The current study indicates that CPR $<1$ continued to develop AEDF in 14 cases, with an average interval of $8.8 \mathrm{~d}$. AEDF progressed to CPR $<1$ in only 3 cases. Moreover, when abnormal umbilical artery blood flow occurs, CPR $<1$ is observed before AEDF in most patients. The TRUFFLE(12) study showed that in addition to minor changes in electronic fetal heart rate, the immediate termination of pregnancy is necessary, and the fetus showing FGR and abnormal umbilical artery blood flow waits until the venous

Page $11 / 16$ 
catheter has an a-wave abnormality to terminate the pregnancy, which may provide an improved longterm outcome. In the current study, only 2 patients were monitored for abnormal a-wave venous catheters while waiting, but these patients suffered intrauterine death within a short period. Therefore, when a significant abnormality of the cord blood flow is detected, it is not appropriate to simply wait for the awave abnormality in the venous catheter to occur before terminating the pregnancy.

After treatment, 4 patients (9.1\%) recovered to normal CPR and continued to monitor the normal cord blood flow until full-term delivery. Therefore, for cases with a fetus of very small gestational age and low birth weight, the survival ability of the newborn after birth and the long-term prognosis are extremely poor; thus, waiting is also a good choice.

Our study compared neonatal outcomes and found poorer outcomes in the group that continued pregnancy than in the group that terminated immediately. The main manifestations indicated that the incidence rates of neonatal asphyxia, neonatal pneumonia, neonatal sepsis, and neonatal anemia were significantly increased, and the incidence rates of neonatal respiratory distress syndrome and bronchopulmonary dysplasia were slightly increased in the continued pregnancy group relative to those in the immediately terminated pregnancy group.

The results of the current study are similar to those in the research conducted by Meher(4) and Flood(5). Their research results showed that the increase in umbilical artery blood flow resistance and blood flow redistribution might not completely achieve brain protection(4). Poor neonatal outcomes, decreased brain volume in neonates, and increased risk of adverse neurocognitive outcomes in children are related to fetal cerebral vasodilation(5). Neurodevelopmental disorders comprise a group of developmental disorders that occur during the developmental period and are often manifested before the child enters elementary school. Developmental disorders and defects lead to impaired personal social, academic, or professional functions. In the current study, motor developmental retardation was assessed using GMS, autism spectrum disorder was detected using M-CHAT, and child development was evaluated using the Gesell scale. Comprehensive assessment of sports, small sports, and social adaptability was also conducted. The aforementioned techniques were applied for the comprehensive assessment of neurodevelopmental disorders in children. The early prognosis of the two groups of children followed in this study showed that the incidence rates of autism spectrum disorder, motor development retardation, and growth retardation were slightly higher in the continued pregnancy group than in the immediately terminated pregnancy group, particularly those with autism spectrum disorder. The rate of neurodevelopmental disorders in children who continue to wait after $\mathrm{CPR}<1$ increased.

Analysis of pregnancy outcome and long-term prognosis of the children in each subgroup of the continued pregnancy group indicated that fetal intrauterine fetal death had a higher probability of occurrence in the patients with persistent CRP $<1$ or patients who further developed AEDF. The incidence of adverse long-term outcomes was higher in children delivered by patients with persistent CRP $<1$ than in the subgroup with normal CRP or CRP that briefly returned to normal during the monitoring period. Therefore, during monitoring of patients with abnormal blood flow in the umbilical artery, those with 
persistent CRP $<1$ or those who further developed AEDF had poor prognosis and should actively terminate pregnancy.

Our study is limited to the loss of some patients to follow-up, which may lead to bias in research results.

\section{Conclusion}

In summary, the increase in umbilical artery blood flow resistance exerts a greater effect on children. CPR $<1$ indicates quite severe intrauterine hypoxia that has been present for a long time. Even if cesarean childbirth is immediately conducted among a large number of children with abnormalities, continuing to wait may cause more neurodevelopment disorders in children. Specifically, patients with persistent CPR < 1 or exhibiting further development of AEDF have poorer prognosis compared with those with CPR $<1$ alone, and pregnancy should be actively terminated. Therefore, if CPR $<1$ occurs, combined with the gestational age, if the fetal survival rate is estimated to be high, the pregnancy should be actively terminated rather than wait until the intravenous catheter becomes A-wave or an abnormal fetal heart rate is observed.

\section{Abbreviations}

cerebroplacental rate (CPR)

absent end-diastolic flow (AEDF)

reversed end-diastolic flow (REDF)

umbilical artery (UA)

middle cerebral artery (MCA)

\section{Declarations}

\section{Acknowledgements}

We would like to thank our patients for cooperating with telephone-follow-up and allowing us to publish their final pregnancy outcome and neonatal outcome.

\section{Authors' contributions}

Hongjuan Ding, Ruizhe Jia, Bimei Hu, Zhaoer Yu, was responsible for the conception of the study and interpretation of findings, Bimei Hu, Zhaoer Yu was responsible for the conception of the study, data analysis and interpretation of findings, and writing of the manuscript. Mingming Gao, Dan Yao, and Yating Qian was responsible for the conception of the study, supervision with data analysis and 
interpretation of findings, and revision of the manuscript. Both authors read and approved the final version of the manuscript.

\section{Funding}

This study was supported by grants from the National Natural Science Foundation of China (No. 81971393, 81771604, 82001546, 81571444), and The Project of Invigorating Health Care through Science, Technology and Education-Jiangsu Provincial Medical Youth Talent (No. QNRC2016112).

\section{Availability of data and materials}

The datasets used and analyzed during the current study are available from the corresponding author on reasonable request.

\section{Ethics approval and consent to participate}

Written consent was obtained from all women, and the study was approved by the Research Ethics Committee of Nanjing Medical University as well as the Nanjing Maternal and Child Health Hospital and the Obstetrics and Gynecology Hospital Affiliated with Nanjing Medical University.

\section{Consent for publication}

Not applicable

\section{Competing interests}

The authors declare that they have no competing interests.

\section{Author details}

${ }^{1}$ Lianyungang Branch of Traditional Chinese Medicine, Jiangsu Union Technical Institute. ${ }^{2}$ Department of obstetrics $₫$ Nanjing Maternal and Child Health HospitalखObstetrics and Gynecology Hospital Affiliated to Nanjing Medical University. ${ }^{3}$ The Fourth School of Clinical Medicine, Nanjing Medical University

\section{References}

1. Giussani DA. The fetal brain sparing response to hypoxia: physiological mechanisms. J Physiol. 2016;594(5):1215-30. 
2. Cahill LS, Hoggarth J, Lerch JP, Seed M, Macgowan CK, Sled JG. Fetal brain sparing in a mouse model of chronic maternal hypoxia. J Cereb Blood Flow Metab. 2019;39(6):1172-84.

3. Oros D, Ruiz-Martinez S, Staines-Urias E, Conde-Agudelo A, Villar J, Fabre E, et al. Reference ranges for Doppler indices of umbilical and fetal middle cerebral arteries and cerebroplacental ratio: systematic review. Ultrasound in obstetrics \& gynecology : the official journal of the International Society of Ultrasound in Obstetrics and Gynecology. 2019;53(4):454-64.

4. Meher S, Hernandez-Andrade E, Basheer SN, Lees C. Impact of cerebral redistribution on neurodevelopmental outcome in small-for-gestational-age or growth-restricted babies: a systematic review. Ultrasound in obstetrics \& gynecology : the official journal of the International Society of Ultrasound in Obstetrics and Gynecology. 2015;46(4):398-404.

5. Flood K, Unterscheider J, Daly S, Geary MP, Kennelly MM, McAuliffe FM, et al. The role of brain sparing in the prediction of adverse outcomes in intrauterine growth restriction: results of the multicenter PORTO Study. American journal of obstetrics and gynecology. 2014;211(3):288.e1-.e5.

6. Simonazzi G, Curti A, Cattani L, Rizzo N, Pilu G. Outcome of severe placental insufficiency with abnormal umbilical artery Doppler prior to fetal viability. BJOG. 2013;120(6):754-7.

7. Caradeux J, Martinez-Portilla RJ, Basuki TR, Kiserud T, Figueras F. Risk of fetal death in growthrestricted fetuses with umbilical and/or ductus venosus absent or reversed end-diastolic velocities before 34 weeks of gestation: a systematic review and meta-analysis. American Journal of Obstetrics and Gynecology. 2018;218(2):S774-S82.e21.

8. ACOG Practice Bulletin No. 204: Fetal Growth Restriction. Obstet Gynecol. 2019;133(2).

9. Conde-Agudelo A, Villar J, Kennedy SH, Papageorghiou AT. Predictive accuracy of cerebroplacental ratio for adverse perinatal and neurodevelopmental outcomes in suspected fetal growth restriction: systematic review and meta-analysis. Ultrasound Obstet Gynecol. 2018;52(4):430-41.

10. Gerber S, Hohlfeld P, Viquerat F, Tolsa JF, Vial Y. Intrauterine growth restriction and absent or reverse end-diastolic blood flow in umbilical artery (Doppler class II or III): A retrospective study of short- and long-term fetal morbidity and mortality. Eur J Obstet Gynecol Reprod Biol. 2006;126(1):20-6.

11. Mose JC. The role of maternal \& fetal doppler in pre-eclampsia. Pregnancy Hypertens. 2014;4(3):242.

12. Lees CC, Marlow N, van Wassenaer-Leemhuis A, Arabin B, Bilardo CM, Brezinka C, et al. 2 year neurodevelopmental and intermediate perinatal outcomes in infants with very preterm fetal growth restriction (TRUFFLE): a randomised trial. Lancet. 2015;385(9983):2162-72.

\section{Figures}




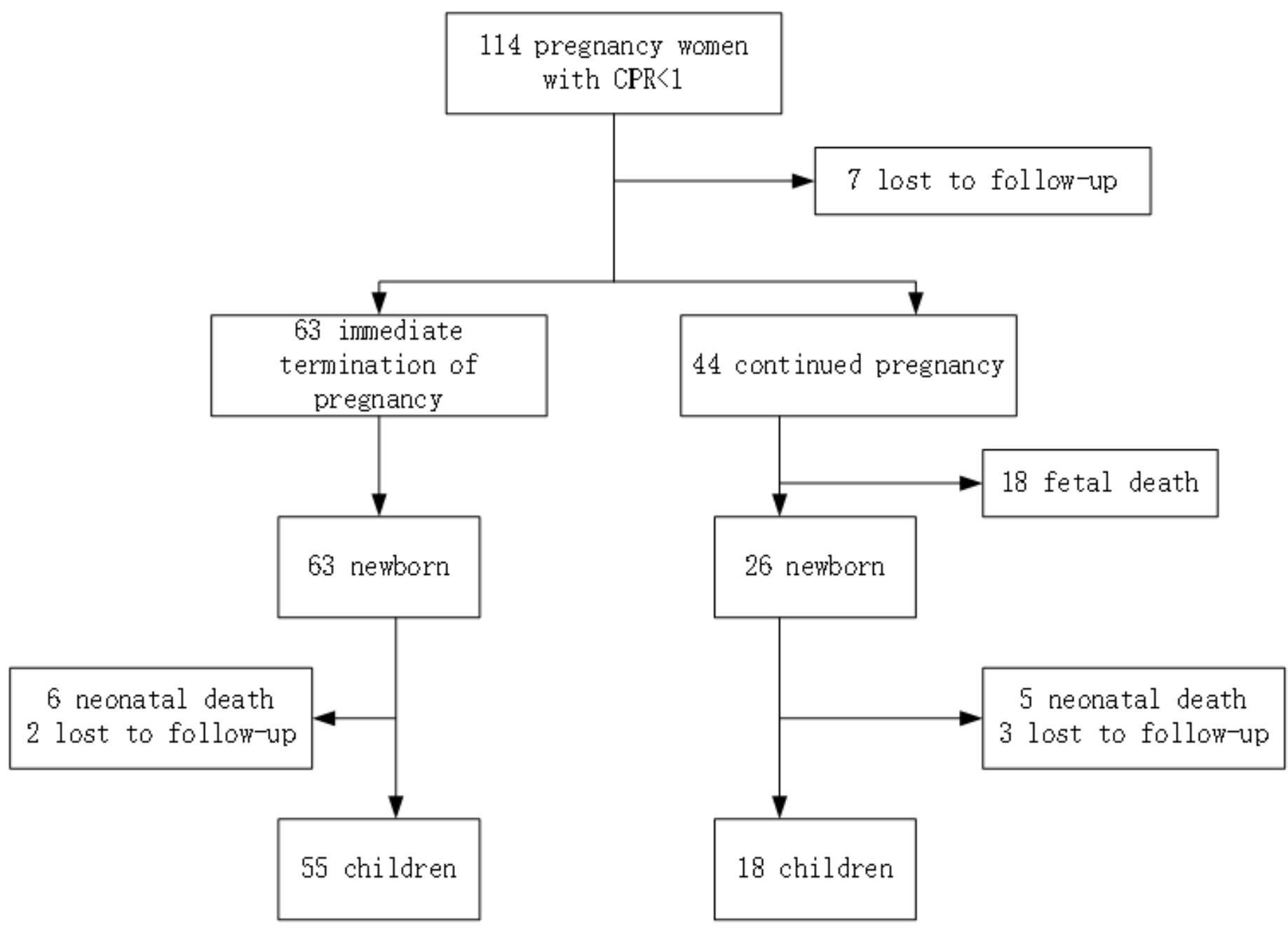

Figure 1

The flowchart of the analysis sample selection 